\title{
用病毒和抑制剂来治疗癌症的组合治疗 数学模型
}

陶有山 (1)*, 郭谦 (2)

(1) 东华大学应用数学系, 上海 200051

(2) 上海师范大学数学系, 上海 200234

*E-mail: taoys@dhu.edu.cn

收稿日期: 2007-11-17; 接受日期: 2008-01-22 国家自然科学基金 (批准号: 10571023) 资助项目

摘要本文考虑一种用肿瘁学上的病毒和抑制剂来治疗癌症的组合治疗方法. 具复制能 力的基因改变的腺病毒感染癌细胞, 在其中复制再生并最终引起感染的癌细胞死亡 (溶解). 一旦感染的癌细胞死亡, 其中的病毒释放并接着感染其他的癌细胞. 病毒能否成功进入癌细 胞与 Coxsackie 腺病毒受体 (CAR) 的表达密切相关. 激活有丝分裂的蛋白质激酶 (即著名的 MEK) 抑制剂能促进 CAR 的表达, 从而导致更多的病毒进入癌细胞. 然而, MEK 抑制剂也 能引起短暂的 G1 细胞循环停止, 从而抑制病毒的复制再生. 为了设计一个有效的治疗方案, 在病毒感染的促进与病毒复制的抑制之间必须取得一个最优平衡. 我们用一个数学模型来 刻画病毒和抑制剂对癌细胞的影响, 并用该模型来探讨: 如何用上述组合治疗方法来促使肿 瘤体积减少. 进一步, 对一定的初始细胞密度, 我们发现了一个最优的抑制剂剂量. 另外, 最 优的抑制剂实施时间也被数值地研究.

关键词肿瘤 肿瘤学上的病毒 MEK 抑制剂 数学建模 $\mathrm{MSC}(2000)$ 主题分类 $35 \mathrm{Q} 80,35 \mathrm{R} 35,92 \mathrm{~A} 15,92 \mathrm{C} 50$

\section{1 引言}

在过去的 30 年里, 许多肿瘤生长的数学模型已被建立; 例如, 参见文献 [1-9] 及其所引用 的参考文献.

化疗广泛用来治疗癌症. 癌症化疗的有效性依赖于到达肿瘤内部的药物浓度. 然而, 癌症 化疗的障碍之一是药物的传送过程. 用于药物传送工具的大分子太大以至于不能把药物传送 到肿瘤内部, 也不能在肿瘤内扩散 ${ }^{[10]}$, 因此, 需要发展其他的治疗方法.

最近发现, 具复制能力的基因改变的肿瘤学上的病毒可以绕过上述传输障碍. 病毒设计 成具有复制能力、有选择的黏附在肿瘤细胞的表面 (但不黏附在正常健康细胞的表面). 然后, 
病毒颗粒通过内吞作用进入肿瘤细胞, 并在肿瘤细胞内复制再生, 最终引起肿瘤细胞死亡 (溶 解). 一旦被感染的癌细胞死亡, 新产生的病毒颗粒释放并继续感染邻近的癌细胞. 这个过程 继续下去直到所有的癌细胞被杀死. 肿瘤的病毒治疗的数学模型最近被建立和研究; 例如, 参 见文献 $[8,9,11]$.

ONYX-015 是一种用于临床医疗实验的肿瘤学上的基因改变的腺病毒. 进一步的医疗 实验证明: Coxsackie 腺病毒受体 (CAR) 的表达对病毒是否成功的进入癌细胞有很大的影 响 ${ }^{[12]}$. 激活有丝分裂的蛋白质激酶 (即著名的 MEK) 抑制剂能促进 CAR 的表达, 从而导致更 多的 ONYX-015 进入癌细胞 ${ }^{[13]}$. 这就产生了一种新奇的用病毒和抑制剂来治疗癌症的组合 治疗方法. 然而, MEK 抑制剂也能引起短暂的细胞循环停止, 从而抑制 ONYX-015 的生命循 环 ${ }^{[13]}$ (细胞循环一般被认为包含 4 个再生增长阶段 (相): 增长相 $\left(\mathrm{G}_{1}\right)$, DNA 合成相 $(\mathrm{S})$, 细 胞分裂前的一段时期 $\left(\mathrm{G}_{2}\right)$ 和有丝分裂相 $(\mathrm{M})$; 和一个拟静止的相 $\left(\mathrm{G}_{0}\right)$. ONYX-015 需要锁 定细胞的 S- 相来进行复制和溶解癌细胞), 因此, MEK 抑制剂可以限制病毒的复制. 为了设 计一个有效的用 ONYX-015 和 MEK 抑制剂来治疗癌症的方案, MEK 抑制剂的正、反两方 面的效果应取得一个最优平衡. 这就使得 MEK 抑制剂、病毒和肿瘤细胞的动力学变得十分 复杂.

本文提出并分析用 ONYX-015 和 MEK 抑制剂来治疗一个球对称肿瘤的数学模型. 模型 包含未被感染的瘤细胞、感染的瘤细胞、坏死的瘤细胞密度, 细胞外病毒密度, Coxsackie 腺 病毒受体 (CAR) 的表达水平和 MEK 抑制剂的强度. 模型是一个非线性偏微分方程系统的自 由边界问题, 其中肿瘤表面为自由边界. 该模型是 Zurakowski 和 Wodarz 的模型 ${ }^{[13]}$ 推广. 他 们的模型假设空间一致性, 因而是一种简化模型. 然而, 具有空间结构的肿瘤需要带空间变量 的偏微分方程模型来刻画. 本文的建模方法是文献 $[9,11,13]$ 中建模方法的综合. 在我们的 $\mathrm{PDE}$ 模型中, 肿瘤组织被视为一个不可压缩的流体, 其中的细胞以速度 $u$ 运动. 局部的细胞 再生和死亡引起压力的变化, 从而导致细胞运动 (根据 Darcy 定律). 本文在抑制剂的强度为 强、弱两种情形下分别找到了肿瘤根除的清晰的生物参数条件. 对一定的初始细胞密度, 我 们也发现了一个最优的抑制剂剂量. 本文也可以看成文献 [11] 中模型的推广: 该模型处理肿 瘤的病毒治疗, 而本文处理肿瘤的病毒和 MEK 抑制剂的组合治疗, 本文允许研究可能的最 优组合治疗方案. 本文还去掉了文献 [11] 中两个关键的技术假设 (10.1) 和 (10.2). 这个推广 是重要的, 因为平衡点 $\left(x_{*}, y_{*}\right)$ 是文献 [11] 中 4 个平衡点中最重要的一个, 而假设 (10.1) 和 (10.2) 生物上又是很严格的.

\section{2 模型}

数学模型包含以下变量:

$\hat{x}=$ 未被感染的肿瘤细胞密度, 即单位体积 $\left(\mathrm{mm}^{3}\right)$ 内未被感染的肿瘤细胞个数,

$\hat{y}=$ 感染的肿瘤细胞密度,

$\hat{n}=$ 坏死的肿瘤细胞密度,

$\hat{v}=S$ 自由病毒密度, 即细胞外的病毒密度,

$\hat{q}=$ 细胞表面 CAR 的表达水平, 
和

$$
u=\text { 肿瘤内细胞的速度. }
$$

肿瘤组织被视为一个不可压缩的流体, 其中的细胞以速度 $u$ 运动. 局部的细胞再生和死亡引 起压力的变化, 从而导致细胞运动 (根据 Darcy 定律). 与细胞的直径相比, 病毒颗粒的直径很 小, 因此, 正如文献 $[11,14,15]$ 那样, 我们只考虑病毒的随机扩散运动. 由于肿瘤是球对称的, 上述未知变量仅依赖于 $(r, t)$, 其中 $r$ 是肿瘤半径. 根据质量守恒律, 在肿瘤区域 $\{r<R(t)\}$ 内模型包含以下方程:

$$
\begin{aligned}
& \frac{\partial \hat{x}(r, t)}{\partial t}+\frac{1}{r^{2}} \frac{\partial}{\partial r}\left[r^{2} u(r, t) \hat{x}(r, t)\right]=\lambda[1-P(t)] \hat{x}(r, t)-\beta \hat{q}(t) \hat{x}(r, t) \hat{v}(r, t), \\
& \frac{\partial \hat{y}(r, t)}{\partial t}+\frac{1}{r^{2}} \frac{\partial}{\partial r}\left[r^{2} u(r, t) \hat{y}(r, t)\right]=\beta \hat{q}(t) \hat{x}(r, t) \hat{v}(r, t)-\delta[1-P(t)] \hat{y}(r, t), \\
& \frac{\partial \hat{n}(r, t)}{\partial t}+\frac{1}{r^{2}} \frac{\partial}{\partial r}\left[r^{2} u(r, t) \hat{n}(r, t)\right]=\delta[1-P(t)] \hat{y}(r, t)-\mu \hat{n}(r, t), \\
& \frac{\partial \hat{v}(r, t)}{\partial t}-D \frac{1}{r^{2}} \frac{\partial}{\partial r}\left[r^{2} \frac{\partial \hat{v}(r, t)}{\partial r}\right]=N \delta[1-P(t)] \hat{y}(r, t)-\gamma \hat{v}(r, t), \\
& \frac{d \hat{q}(t)}{d t}=\eta P(t)[a-\hat{q}(t)] .
\end{aligned}
$$

MEK 抑制剂强度用一个参数 $P$ 来刻画 ${ }^{[13]}$. 为了能用模型来研究可能的抑制剂 “最优实施 时间”, 我们这里假设 $P=P(t)$, 它的值介于 0 和 1 之间. 我们用 $\hat{q}$ 来定义细胞表面 CAR 的 平均表达水平, 它受 MEK 抑制剂的调控, 因此, $\hat{q}=\hat{q}(t)$ 是空间一致的. 在 (1) 式中, $\lambda$ 是在 没有应用 MEK 抑制剂时的未被感染的细胞的 “净再生率” (= 出生率 - 死亡率). 我们忽略 外部营养的效果, 假定所有的细胞在没有应用 MEK 抑制剂时的 “净再生率” 为常数 (参见文 献 $[6,9,11,13-21]$ ). MEK 抑制剂能降低未被感染细胞的再生, 其效果用 $1-P(t)$ (参见文献 [13]) 来表示. 当病毒遇到未被感染的细胞时, 感染就发生了, 病毒黏附着未被感染的瘤细胞 表面的 CAR 受体. 感染率与瘤细胞表面的 CAR 受体的数目 $\hat{q}$ 、细胞外的病毒密度 $\hat{v}$ 和未 被感染的肿瘤细胞密度 $\hat{x}$ 成正比. $\beta$ 是感染的速率 ${ }^{[9,13]}$. 在 (2) 式中, $\delta$ 是感染的细胞的死

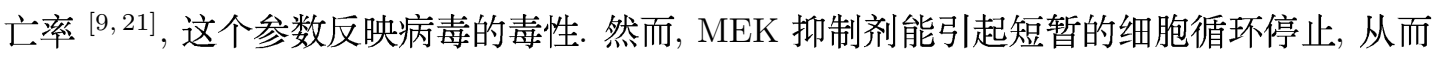
抑制病毒的生命循环和病毒引起的细胞死亡 (病毒需要锁定细胞的 S- 相来溶解细胞). 所以, 病毒引起的细胞死亡率和 $1-P(t)$ 成正比 ${ }^{[13]}$. 在 (3) 式中, $\mu$ 是坏死细胞的清除率 ${ }^{[9,11]}$. 在 (4) 式中, $\gamma$ 是病毒的清除率 ( $1 / \gamma$ 是病毒的平均寿命), $D$ 是病毒的扩散系数 $[11,14,15,18,20]$, $N \delta$ 是在没有应用 MEK 抑制剂的情形下病毒的释放率 $(N$ 是在一个瘤细胞死亡时所释放的 病毒数目 ${ }^{[9,11]}$. 如前面所述, MEK 抑制剂能引起短暂的细胞循环停止, 从而抑制病毒的生命 循环 (病毒需要锁定细胞的 S- 相来进行复制). 因此, 在应用 MEK 抑制剂的情形下病毒的释 放率会降低 $\left(\right.$ 与 $1-P(t)$ 成正比 ${ }^{[13]}$. 在 $(5)$ 式中, 细胞以速率 $\eta$ 产出 CAR 受体, 产出率受 MEK 抑制剂强度 $P$ 调控. 表达式 $a-\hat{q}$ 表示 CAR 受体表达的饱和, 即瘤细胞不能无限的产 出 CAR 受体 (参见文献 [13]).

我们假设肿瘤组织内没有空隙 $[6,7,9,11,14,16,21]$, 因此

$$
\hat{x}+\hat{y}+\hat{n} \equiv \text { 常数 } \equiv \theta \text {. }
$$

将方程 (1)-(3) 相加并利用假设 (6) 推得

$$
\frac{\theta}{r^{2}} \frac{\partial}{\partial r}\left(r^{2} u(r, t)\right)=\lambda[1-P(t)] \hat{x}(r, t)-\mu \hat{n}(r, t) .
$$


假设病毒不跑出肿瘤, 因此

$$
\frac{\partial}{\partial r} \hat{v}(0, t)=0, \quad \frac{\partial}{\partial r} \hat{v}(R(t), t)=0,
$$

其中第 1 个方程源于球对称假设.

最后, 肿瘤表面的速度等于那里的细胞运动速度:

$$
\frac{d R(t)}{d t}=u(R(t), t) .
$$

注意到 (3) 式可由 (1)、(2)、(6) 和 (7) 式推得, 所以, 在后面将丢掉这个方程, 并在 (7) 式 中用 $\theta-\hat{x}-\hat{y}$ 来代替 $\hat{n}$.

我们也注意到速度是对称的:

$$
u(0, t)=0
$$

为完成自由边界问题的陈述, 我们提出如下初始条件:

$$
\left\{\begin{array}{l}
R(0) \text { 给定, } \\
\hat{x}(r, 0)=\hat{x}_{0}(r), \quad \hat{y}(r, 0)=\hat{y}_{0}(r), \quad \hat{v}(r, 0)=\hat{v}_{0}(r), \quad \hat{q}(0)=\hat{q}_{0} \geqslant 0,
\end{array}\right.
$$

这里 $\hat{x}_{0}(r), \hat{y}_{0}(r)$ 和 $\hat{v}_{0}(r)$ 是非负函数, 满足 $\hat{x}_{0}(r)+\hat{y}_{0}(r) \leqslant \theta$, 其中 $0 \leqslant r \leqslant R(0)$.

注 2.1 如果 $P(t) \equiv 0$, 那么由 $(5)$ 式有

$$
\hat{q}(t) \equiv \text { 常数 }(>0) \text {. }
$$

这时模型 (1)-(11) 就是文献 $[9,11]$ 中的模型, 因此, 模型 (1)-(11) 可看成文献 [9, 11] 中模型 的推广. 模型 (1)-(11) 处理肿瘤的病毒和 MEK 抑制剂的组合治疗, 而文献 $[9,11]$ 的模型处 理肿瘤的单一的病毒治疗. 在第 8 节, 我们将看到组合治疗的效果好于单独用病毒来进行治 疗.

下一节, 我们将自由边界问题 (1)-(11) 变换成固定区域上的问题.

\section{3 变换}

引进变量

$$
\tilde{x}=\frac{\hat{x}}{\theta}, \quad \tilde{y}=\frac{\hat{y}}{\theta}, \quad \tilde{v}=\frac{\hat{v}}{\theta N}, \quad \tilde{q}=\hat{q}, \quad \tilde{u}=u
$$

和数量

$$
p_{0}=\frac{\beta N \theta}{\gamma},
$$

其中参数 $p_{0}$ 表示一个病毒平均释放的病毒数目 (参见文献 [9]).

根据新变量, 系统 (1)-(11) 在 $\{r<R(t), t>0\}$ 中变成如下形式:

$$
\begin{aligned}
& \frac{d \tilde{q}}{d t}=\eta P(t)(a-\tilde{q}), \quad \tilde{q}(0)=\tilde{q}_{0} \geqslant 0, \\
& \frac{\partial \tilde{x}}{\partial t}=\lambda[1-P(t)] \tilde{x}-p_{0} \gamma \tilde{q} \tilde{x} \tilde{v}-\frac{1}{r^{2}} \frac{\partial}{\partial r}\left(r^{2} \tilde{u} \tilde{x}\right), \\
& \frac{\partial \tilde{y}}{\partial t}=p_{0} \gamma \tilde{q} \tilde{x} \tilde{v}-\delta[1-P(t)] \tilde{y}-\frac{1}{r^{2}} \frac{\partial}{\partial r}\left(r^{2} \tilde{u} \tilde{y}\right), \\
& \tilde{x}(r, 0)=\tilde{x}_{0}(r), \quad \tilde{y}(r, 0)=\tilde{y}_{0}(r), \quad \tilde{x}_{0}(r), \tilde{y}_{0}(r) \geqslant 0, \quad \tilde{x}_{0}(r)+\tilde{y}_{0}(r) \leqslant 1,
\end{aligned}
$$




$$
\begin{aligned}
& \frac{\partial \tilde{v}}{\partial t}-\frac{D}{r^{2}} \frac{\partial}{\partial r}\left(r^{2} \frac{\partial \tilde{v}}{\partial r}\right)=\delta[1-P(t)] \tilde{y}-\gamma \tilde{v}, \\
& \frac{\partial}{\partial r} \tilde{v}(0, t)=0, \quad \frac{\partial}{\partial r} \tilde{v}(R(t), t)=0, \\
& \tilde{v}(r, 0)=\tilde{v}_{0}(r) \geqslant 0, \\
& \frac{1}{r^{2}} \frac{\partial}{\partial r}\left(r^{2} \tilde{u}\right)=\lambda[1-P(t)] \tilde{x}-\mu(1-\tilde{x}-\tilde{y}), \\
& \tilde{u}(0, t)=0, \\
& \frac{d R(t)}{d t}=\tilde{u}(R(t), t), \quad t>0, \quad R(0) \text { 给定. }
\end{aligned}
$$

引进新的变量

$$
\rho=\rho(r, t)=\frac{r}{R(t)}
$$

$$
\begin{aligned}
& x(\rho, t)=\tilde{x}(r, t), \quad y(\rho, t)=\tilde{y}(r, t), \quad v(\rho, t)=\tilde{v}(r, t), \\
& q(t)=\tilde{q}(t), \quad u(\rho, t)=\tilde{u}(r, t) / R(t),
\end{aligned}
$$

系统 (12)-(21) 在区域 $\{0<\rho<1, t>0\}$ 中变成以下形式:

$$
\begin{aligned}
& \frac{d q}{d t}=\eta P(t)(a-q), \quad q(0)=q_{0} \geqslant 0, \\
& \frac{\partial x}{\partial t}+[u(\rho, t)-\rho u(1, t)] \frac{\partial x}{\partial \rho} \\
& \quad=\lambda[1-P(t)] x-p_{0} \gamma q x v-[-\mu+(\lambda+\mu-\lambda P(t)) x+\mu y] x, \\
& \frac{\partial y}{\partial t}+[u(\rho, t)-\rho u(1, t)] \frac{\partial y}{\partial \rho} \\
& \quad=p_{0} \gamma q x v-\delta[1-P(t)] y-[-\mu+(\lambda+\mu-\lambda P(t)) x+\mu y] y, \\
& x(\rho, 0)=x_{0}(\rho), \quad y(\rho, 0)=y_{0}(\rho), \quad x_{0}(\rho), y_{0}(\rho) \geqslant 0, \quad x_{0}(\rho)+y_{0}(\rho) \leqslant 1, \\
& \frac{\partial v}{\partial t}-\frac{D}{R^{2}(t)} \frac{1}{\rho^{2}} \frac{\partial}{\partial \rho}\left(\rho^{2} \frac{\partial v}{\partial \rho}\right)-\rho u(1, t) \frac{\partial v}{\partial \rho}=\delta[1-P(t)] y-\gamma v, \\
& v_{\rho}(0, t)=0, \quad v_{\rho}(1, t)=0, \\
& v(\rho, 0)=v_{0}(\rho) \geqslant 0, \\
& u(\rho, t)=\frac{1}{\rho^{2}} \int_{0}^{\rho} s^{2}\{-\mu+[\lambda+\mu-\lambda P(t)] x(s, t)+\mu y(s, t)\} d s, \\
& \dot{R}(t)=R(t) u(1, t), \quad R(0) \text { 给定. }
\end{aligned}
$$

我们将假设

$$
x_{0}(\rho), y_{0}(\rho), v_{0}(\rho) \in C^{1}[0,1] \text { 且 } \frac{\partial v_{0}}{\partial \rho}(1)=0 .
$$

正如文献 [11] 中定理 2.1 的证明, 我们能得到如下的关于系统 (22)-(31) 的整体解的存 在唯一性结果: 
定理 3.1 系统 (22)-(31) 有唯一解 $(q(t), x(\rho, t), y(\rho, t), v(\rho, t), u(\rho, t), R(t))$ 且 $x, \partial x / \partial \rho$, $y, \partial y / \partial \rho, v, \partial v / \partial \rho$, 和 $u, \partial u / \partial \rho$ 属于 $C[0 \leqslant \rho \leqslant 1,0<t<\infty], q(t)$ 和 $R(t)$ 属于 $C^{1}[0, \infty)$,

$$
R(0) e^{-\alpha t}<R(t)<R(0) e^{\alpha t}
$$

其中 $\alpha$ 为某个正常数.

在第 4-7 节我们将寻找肿瘤根除的清晰的参数条件.

\section{4 一致平衡解}

本节假定

$$
P(t) \equiv \text { 常数 }=P \text {. }
$$

由 (29) 和 $(30)$ 式推得

$$
\frac{d R(t)}{d t}=R(t) \int_{0}^{1} s^{2}[-\mu+(\lambda+\mu-\lambda P) x(s, t)+\mu y(s, t)] d s .
$$

一旦 $x$ 和 $y$ 达到它们的平衡态, (32) 式中 $\frac{d R(t)}{d t}$ 的符号就不变. 因此, 肿瘤的长期行为可以通 过简单的分析 (22)-(24) 和 (26) 式的一致平衡解 $E_{s}=\left(x_{s}, y_{s}, v_{s}, q_{s}\right)$ 来预见, 这些一致平衡 解满足如下方程:

$$
\begin{aligned}
& \eta P\left(a-q_{s}\right)=0, \\
& \lambda(1-P) x_{s}-p_{0} \gamma q_{s} x_{s} v_{s}-\left[-\mu+(\lambda+\mu-\lambda P) x_{s}+\mu y_{s}\right] x_{s}=0, \\
& p_{0} \gamma q_{s} x_{s} v_{s}-\delta(1-P) y_{s}-\left[-\mu+(\lambda+\mu-\lambda P) x_{s}+\mu y_{s}\right] y_{s}=0, \\
& \delta(1-P) y_{s}-\gamma v_{s}=0 .
\end{aligned}
$$

记

$$
\bar{p}_{0}=p_{0} a, \quad \bar{P}=1-P .
$$

容易发现, 有 4 个一致平衡解

$$
\begin{aligned}
& E_{1}=(0,0,0, a), \\
& E_{2}=(1,0,0, a), \\
& E_{3}=\left(0,1-\frac{\delta}{\mu} \bar{P}, \frac{\delta}{\gamma} \bar{P}\left(1-\frac{\delta}{\mu} \bar{P}\right), a\right), \quad \text { 假定 } \delta \bar{P}<\mu, \\
& E_{4}=\left(x_{*}, y_{*}, \frac{\delta}{\gamma} \bar{P} y_{*}, a\right),
\end{aligned}
$$

其中

$$
x_{*}=\frac{\lambda \mu-\bar{p}_{0} \delta \mu+\bar{p}_{0} \delta^{2} \bar{P}+\mu \delta}{\left(\bar{p}_{0} \delta-\lambda\right) \bar{p}_{0} \delta \bar{P}}, \quad y_{*}=\frac{(\lambda \bar{P}+\mu)\left(\bar{p}_{0} \delta-\delta-\lambda\right)}{\left(\bar{p}_{0} \delta-\lambda\right) \bar{p}_{0} \delta \bar{P}},
$$

假定 $x_{*} \geqslant 0, y_{*} \geqslant 0$.

由 (33)-(36) 式, 我们发现: $\left(x_{*}, y_{*}\right)$ 满足

$$
\left\{\begin{array}{l}
\lambda \bar{P}+\mu-(\lambda \bar{P}+\mu) x_{*}-\left(\bar{p}_{0} \delta \bar{P}+\mu\right) y_{*}=0 \\
\mu-\delta \bar{P}+\left(\bar{p}_{0} \delta \bar{P}-\lambda \bar{P}-\mu\right) x_{*}-\mu y_{*}=0
\end{array}\right.
$$


后面将会用到上述条件. 在我们对平衡解 $E_{4}$ 的稳定性分析中, 假设

$$
\bar{p}_{0}>1+\frac{\lambda}{\mu} \bar{P}+\frac{\lambda}{\delta},
$$

对文献 $[9,21]$ 中给出的典型参数值和 $0 \leqslant \bar{P} \leqslant 1$, 上述条件成立.

最后说明: 如果 (44) 式成立且

$$
\delta \bar{P} \geqslant \mu
$$

那么由 (42) 式有

$$
x_{*}>0, \quad y_{*}>0 .
$$

\section{5 坏死肿瘤和未治疗肿瘤的稳定性}

对平衡解 $E_{1}$, 我们有 $n_{s} \equiv 1-x_{s}-y_{s}=1$, 因此 $E_{1}$ 对应于坏死肿瘤; 对平衡解 $E_{2}$, 我们 有 $x_{s}=1$ 和 $y_{s}=v_{s}=0$, 因此 $E_{2}$ 对应于未治疗肿瘤. 本节将讨论平衡解 $E_{1}$ 和 $E_{2}$ 的稳定 性. 将 (23) 和 (24) 式的右端项分别记为 $f_{1}(x, y, v, q)$ 和 $f_{2}(x, y, v, q)$. 通过直接计算得到:

$$
\begin{aligned}
& \left.\frac{\partial f_{1}}{\partial x}\right|_{E_{s}}=\lambda \bar{P}+\mu-2(\lambda \bar{P}+\mu) x_{s}-\mu y_{s}-\bar{p}_{0} \delta \bar{P} y_{s}, \\
& \left.\frac{\partial f_{1}}{\partial y}\right|_{E_{s}}=-\mu x_{s}, \\
& \left.\frac{\partial f_{2}}{\partial x}\right|_{E_{s}}=\bar{p}_{0} \delta \bar{P} y_{s}-(\lambda \bar{P}+\mu) y_{s}, \\
& \left.\frac{\partial f_{2}}{\partial y}\right|_{E_{s}}=-\left[\delta \bar{P}-\mu+(\lambda \bar{P}+\mu) x_{s}+2 \mu y_{s}\right] .
\end{aligned}
$$

先考虑平衡解 $E_{1}$. 由 (46)-(49) 式, 双曲系统 (23) 和 (24) 的右端项在平衡点 $E_{1}$ 处的线 性化系数矩阵 $A_{1}$ 有如下形式:

$$
A_{1}=\left(\begin{array}{cc}
\frac{\partial f_{1}}{\partial x} & \frac{\partial f_{1}}{\partial y} \\
\frac{\partial f_{2}}{\partial x} & \frac{\partial f_{2}}{\partial y}
\end{array}\right)_{E_{1}}=\left(\begin{array}{cc}
\lambda \bar{P}+\mu & 0 \\
0 & \mu-\delta \bar{P}
\end{array}\right),
$$

显然, $A_{1}$ 至少有一个正特征值 $\lambda \bar{P}+\mu$. 所以有

\section{定理 5.1 平衡解 $E_{1}$ 线性不稳定.}

注 5.1 数学上, 平衡解 $E_{1}$ 的线性不稳定隐含: 存在 $\varepsilon_{0}>0$, 对任意的小的正常数 $\delta>0$, 存在某个满足 $\|x(\cdot, 0)\|_{C[0,1]}+\|y(\cdot, 0)\|_{C[0,1]}<\delta$ 的初始条件 $(x(\rho, 0), y(\rho, 0))$, 使得系统 $(22)-(31)$ 的解 $(x(\rho, t), y(\rho, t))$ 满足

$$
\|x(\cdot, t)\|_{C[0,1]}+\|y(\cdot, t)\|_{C[0,1]} \geqslant \varepsilon_{0}, \quad \text { 当 } t \rightarrow+\infty .
$$

然而, “ $\|x(\cdot, t)\|_{C[0,1]}+\|y(\cdot, t)\|_{C[0,1]} \geqslant \varepsilon_{0}$, 当 $t \rightarrow+\infty$ ” 生物上意味着: 当 $t$ 充分大后, 坏死的 肿瘤复活.

下面研究平衡解 $E_{2}$ 的稳定性. 由 (46)-(49) 式, 双曲系统 (23) 和 (24) 的右端项在平衡点 $E_{2}$ 处的线性化系数矩阵 $A_{2}$ 有如下形式:

$$
A_{2}=\left(\begin{array}{cc}
\frac{\partial f_{1}}{\partial x} & \frac{\partial f_{1}}{\partial y} \\
\frac{\partial f_{2}}{\partial x} & \frac{\partial f_{2}}{\partial y}
\end{array}\right)_{E_{2}}=\left(\begin{array}{cc}
-(\lambda \bar{P}+\mu) & -\mu \\
0 & -(\lambda+\delta) \bar{P}
\end{array}\right) .
$$


定理 5.2 假定

$$
0 \leqslant P<1,
$$

则系统 (22)-(31) 的平衡解 $E_{2}$ 是局部稳定的.

证明 在假设 (50) 下, 矩阵 $A_{2}$ 的两个特征值是负的. 因此, 系统 (22)-(31) 的平衡解 是线性稳定的. 进一步, 类似文献 [11] 中引理 6.2 , 定理 7.1 和 8.1 的证明, 我们能得到系统 (22)-(31) 的平衡解是非线性稳定的. 证毕.

注 5.2 定理 5.2 隐含, 如果 MEK 抑制剂的强度不是太强, 那么没有被注射病毒的肿瘤 将持续增长. 事实上, 由 (29) 和 (30) 式及定理 5.2 有

$$
\begin{aligned}
\frac{\dot{R}(t)}{R(t)} & =\int_{0}^{1} s^{2}[-\mu+(\lambda \bar{P}+\mu)+O(\varepsilon)] d s \\
& =\frac{\lambda \bar{P}}{3}+O(\varepsilon),
\end{aligned}
$$

其中 $\varepsilon$ 充分小. 因此,

$$
R(t)=R(0) e^{\left(\frac{\lambda \bar{P}}{3}+O(\varepsilon)\right) t} \rightarrow+\infty, \text { 当 } t \rightarrow+\infty .
$$

然而, 如果 MEK 的强度很强: $P=1$, 那么细胞循环停止, 从而瘤细胞的再生停止, 肿瘤也停 止生长. 事实上, 如果 $P=1$, 那么由 $(29)$ 和 $(30)$ 式及 $x(\rho, t)+y(\rho, t) \leqslant 1$ 有

$$
\frac{\dot{R}(t)}{R(t)}=\int_{0}^{1} s^{2}[-\mu+\mu(x(s, t)+y(s, t))] d s \leqslant 0,
$$

即

$$
\dot{R}(t) \leqslant 0
$$

\section{6 强抑制剂情形: $\delta(1-P)<\mu$}

对文献 [21] 中给出的参数值: $\delta=\frac{1}{48} \mathrm{hr}^{-1}$ 和 $\mu=\frac{1}{72} \mathrm{hr}^{-1}$, 我们有

$$
\delta>\mu \text {. }
$$

本节假设抑制剂的强度满足

$$
\delta(1-P)<\mu \Leftrightarrow \delta \bar{P}<\mu .
$$

在假设 (52) 下, 平衡解 $E_{3}$ 存在. 本节研究平衡解 $E_{3}$ 的稳定性. 由 (46)-(49) 式, 双曲系统 (23) 和 (24) 的右端项在平衡点 $E_{3}$ 处的线性化系数矩阵 $A_{3}$ 有如下形式:

$$
A_{3}=\left(\begin{array}{cc}
\frac{\partial f_{1}}{\partial x} & \frac{\partial f_{1}}{\partial y} \\
\frac{\partial f_{2}}{\partial x} & \frac{\partial f_{2}}{\partial y}
\end{array}\right)_{E_{3}}=\left(\begin{array}{cc}
(\lambda+\delta) \bar{P}-\bar{p}_{0} \delta \bar{P}\left(1-\frac{\delta}{\mu} \bar{P}\right) & 0 \\
\left(\bar{p}_{0} \delta \bar{P}-\lambda \bar{P}-\mu\right)\left(1-\frac{\delta}{\mu} \bar{P}\right) & -(\mu-\delta \bar{P})
\end{array}\right) .
$$

除 (52) 式之外, 本节还假设

$$
\bar{p}_{0}>\frac{(\lambda+\delta) \mu}{\delta(\mu-\delta \bar{P})} .
$$

显然, 在假设 (52) 和 (53) 之下, 矩阵 $A_{3}$ 的两个特征值是负的. 因此, 系统 (22)-(31) 的平衡 解 $E_{3}$ 是线性稳定的. 进一步, 类似文献 [11] 中引理 6.2 , 定理 7.1 和 8.1 的证明, 我们能得到 系统 (22)-(31) 的平衡解 $E_{3}$ 是非线性稳定的. 更确切地说, 我们有 (参见文献 [11, 定理 9.1])

定理 6.1 假设 (52) 和 (53) 式成立, 并设 $R(0)$ 为任意的正数. 如果 


$$
\left\|x_{0}(\rho), y_{0}(\rho)-\left(1-\frac{\delta}{\mu} \bar{P}\right), v_{0}(\rho)-\frac{\delta}{\gamma} \bar{P}\left(1-\frac{\delta}{\mu} \bar{P}\right)\right\|_{C^{1}[0,1]}
$$

充分小, 则对所有的 $t>0$, 成立 $\dot{R}(t)<0$ 且

$$
R(t) \leqslant R(0) e^{-\alpha t} \text { 当 } t \rightarrow \infty \text {, 对某个 } \alpha>0 .
$$

定理 6.1 建议: 无论初始的肿瘤是多么大, 如果初始的感染的瘤细胞密度接近 $\left(1-\frac{\delta}{\mu} \bar{P}\right)$, 而未被感染的瘤细胞密度充分小, 那么注射密度接近 $\frac{\delta}{\gamma} \bar{P}\left(1-\frac{\delta}{\mu} \bar{P}\right)$ 的病毒和强度接近 $P$ 的 MEK 抑制剂后, 就能使肿瘤体积以指数速率减少.

注 6.1 定理 6.1 中假设 $R(0)$ 为任意正数. 这个假设仅在数学意义上成立. 事实上, 如 果初始肿瘤太大, 实际生物世界中的肿瘤宿主不可能存活. 因此, “任意正数” 应理解为 “合理 范围内的任意正数”.

注 6.2 对 $q_{0} \geqslant 0$ 和 $P(t) \equiv P>0$, 由 (22) 式有

$$
q(t)=a+\left(q_{0}-a\right) e^{-\eta P t} \rightarrow a, \text { 当 } t \rightarrow+\infty .
$$

注 6.3 参数条件 (53) 隐含: 为减少肿瘤体积, 对病毒复制能力的要求强烈的依赖于 MEK 抑制剂的强度, 即 MEK 抑制剂的活性越强, 对病毒复制能力的要求就越低 (这可能是 由于更高的病毒感染率).

\section{7 弱抑制剂情形: $\delta(1-P) \geqslant \mu$}

对文献 [21] 中给定的参数 $\delta$ 和 $\mu$, 不等式 (51) 成立. 本节假设 MEK 抑制剂的活性不太 强, 使得下式成立:

$$
\delta(1-P) \geqslant \mu \Leftrightarrow \delta \bar{P} \geqslant \mu .
$$

为研究平衡解 $E_{4}$ 的稳定性, 本节假设

$$
\bar{p}_{0}>1+\frac{\lambda}{\mu} \bar{P}+\frac{\lambda}{\delta} .
$$

由 (46)-(49) 和 (43) 式发现, 双曲系统 (23) 和 (24) 的右端项在平衡点 $E_{4}$ 处的线性化系数矩 阵 $A_{4}$ 有如下形式:

$$
A_{4}=\left(\begin{array}{cc}
\frac{\partial f_{1}}{\partial x} & \frac{\partial f_{1}}{\partial y} \\
\frac{\partial f_{2}}{\partial x} & \frac{\partial f_{2}}{\partial y}
\end{array}\right)_{E_{4}}=\left(\begin{array}{cc}
-(\lambda \bar{P}+\mu) x_{*} & -\mu x_{*} \\
\left(\bar{p}_{0} \delta \bar{P}-\lambda \bar{P}-\mu\right) y_{*} & -\bar{p}_{0} \delta \bar{P} x_{*}-\mu y_{*}
\end{array}\right) .
$$

由 (42), (55) 和 (56) 式容易验证

$$
x_{*}>0, \quad y_{*}>0
$$

从而

$$
\begin{aligned}
\operatorname{Tr} A_{4} & =-\left(\bar{p}_{0} \delta \bar{P}+\lambda \bar{P}+\mu\right) x_{*}-\mu y_{*}<0, \\
\left|A_{4}\right| & =(\lambda \bar{P}+\mu) x_{*}\left(\bar{p}_{0} \delta \bar{P} x_{*}+\mu y_{*}\right)+\mu x_{*}\left(\bar{p}_{0} \delta \bar{P}-\lambda \bar{P}-\mu\right) y_{*} \\
& =(\lambda \bar{P}+\mu) \bar{p}_{0} \delta \bar{P} x_{*}^{2}+\mu \bar{p}_{0} \delta \bar{P} x_{*} y_{*}>0 .
\end{aligned}
$$

由 (58) 和 (59) 式推得矩阵 $A_{4}$ 的两个特征值具有负实部, 因此系统 (22)-(31) 的平衡解 $E_{4}$ 是线性稳定的. 进一步, 类似文献 [11] 中引理 6.2、定理 7.1 和 8.1 的证明, 我们能得到系统 (22)-(31) 的平衡解 $E_{4}$ 是非线性稳定的. 更确切地说 (参见文献 [11, 定理 9.1]) 
定理 7.1 假设 $(55)$ 和 $(56)$ 式成立, 并设 $R(0)$ 为任意正数. 如果

$$
\left\|x_{0}(\rho)-x_{*}, y_{0}(\rho)-y_{*}, v_{0}(\rho)-\frac{\delta}{\gamma} \bar{P} y_{*}\right\|_{C^{1}[0,1]}
$$

充分小, 那么对所有的 $t>0$, 成立 $\dot{R}(t)<0$ 且

$$
R(t) \leqslant R(0) e^{-\alpha t} \text { 当 } t \rightarrow \infty \text {, 对某个 } \alpha>0 \text {. }
$$

证明 由 (29) 和 (30) 式以及平衡解 $E_{4}$ 的局部非线性稳定性, 我们推得

$$
\begin{aligned}
\frac{\dot{R}(t)}{R(t)} & =\int_{0}^{1} s^{2}[-\mu+(\lambda \bar{P}+\mu) x(s, t)+\mu y(s, t)] d s \\
& =\int_{0}^{1} s^{2}\left[-\mu+(\lambda \bar{P}+\mu) x_{*}+\mu y_{*}+\varepsilon\right] d s \quad(\varepsilon \text { 充分小) } \\
& \left.=-\frac{\delta \mu}{3\left(\bar{p}_{0} \delta-\lambda\right)}\left(\bar{p}_{0}-1-\frac{\lambda}{\mu} \bar{P}-\frac{\lambda}{\delta}\right)+\frac{1}{3} \varepsilon \quad \text { (对充分大的 } t\right) \\
& \leqslant-\frac{\delta \mu}{6\left(\bar{p}_{0} \delta-\lambda\right)}\left(\bar{p}_{0}-1-\frac{\lambda}{\mu} \bar{P}-\frac{\lambda}{\delta}\right):=-\alpha<0 \quad \text { (由 (56) 式). }
\end{aligned}
$$

证毕.

定理 7.1 建议: 无论初始的肿瘤是多么大, 如果初始的感染的瘤细胞密度接近 $y_{*}$, 而未被 感染的瘤细胞密度接近 $x_{*}$, 那么注射密度接近 $\frac{\delta}{\gamma} \bar{P} y_{*}$ 的病毒和强度接近 $P$ 的 MEK 抑制剂 后, 就能使肿瘤体积以指数速率减少.

注 7.1 定理 7.1 推广了文献 [11] 中定理 10.1 的结果: 新的结果包含了 MEK 抑制剂 的应用. 进一步, 本文去掉了文献 [11] 中的两个技术性的参数假设 (10.1) 和 (10.2). 这个推广 是重要的, 因为平衡点 $\left(x_{*}, y_{*}\right)$ 是文献 [11] 中 4 个平衡点中最重要的一个, 而假设 (10.1) 和 (10.2) 在生物上又是很严格的.

\section{8 抑制剂的最优剂量}

先陈述一个关于抑制剂的最优剂量的一个解析结果.

定理 8.1 假设 (55) 和 (56) 式成立, 并进一步假设初始的感染的瘤细胞密度接近 $y_{*}$, 而 未被感染的瘤细胞密度接近 $x_{*}$, 注射的病毒密度接近 $\frac{\delta}{\gamma} \bar{P} y_{*}$ (这里 $\left(x_{*}, y_{*}\right)$ 由 $(42)$ 式给出, 其 中 $\bar{P}=\mu / \delta)$, 则

$$
P_{\text {optimal }}:=1-\frac{\mu}{\delta}
$$

是用病毒和 MEK 抑制剂来治疗肿瘤的一个最优的抑制剂剂量.

证明 在假设 (55) 和 (56) 之下, 定理 7.1 已建立了平衡解 $E_{4}$ 的局部非线性稳定性. 因 此, 我们仅需对 $(x(\rho, t), y(\rho, t), v(\rho, t), q(t)) \equiv E_{4}$ 来证明定理 8.1.

定义数目 $P$ 的一个集合

$$
\Theta=\{0 \leqslant P \leqslant 1 \mid P \text { 满足假设 (55) 和 (56) }\} .
$$

对文献 [21] 中给定的典型参数值 $\delta=\frac{1}{48} \mathrm{hr}^{-1}, \mu=\frac{1}{72} \mathrm{hr}^{-1}, \lambda=3.2 \times 10^{-4} \mathrm{hr}^{-1}, p_{0}=3.73$ 和 文献 [13] 中给定的 $a=2$, 我们有 $0 \in \Theta$, 因此, $\Theta$ 是非空的. 
注意: 对任何给定的 $P \in \Theta$, 存在唯一的由 (41) 和 (42) 给出的平衡解 $E_{4}$, 并由定理 7.1 知它对应于肿瘤的成功治疗. 这个平衡解 $E_{4}$ 随 $P \in \Theta$ 的变化而变化, 由 (29)、(30) 和 (42) 式推得

$$
\begin{aligned}
\frac{\dot{R}(t)}{R(t)} & =\frac{1}{3}\left[-\mu+(\lambda \bar{P}+\mu) x_{*}+\mu y_{*}\right] \\
& =-\frac{\delta \mu}{3\left(\bar{p}_{0} \delta-\lambda\right)}\left(\bar{p}_{0}-1-\frac{\lambda}{\mu} \bar{P}-\frac{\lambda}{\delta}\right) .
\end{aligned}
$$

由假设 (56) 有

$$
\left.\dot{R}(t)\right|_{P \in \Theta}<0 .
$$

既然肿瘤治疗的效果可根据 $R(t)$ 来评估, 且由 (62) 式知 $R(t)$ 又随 $P \in \Theta$ 而变化 (这里假设 (62) 式中其他的参数是给定的), 我们提出下列问题:

是否有一个 $P_{*} \in \Theta$, 使得

$$
\left.\left.R(t)\right|_{P=P_{*}}=\min _{P \in \Theta} R(t) \quad \text { (对大的 } t\right)
$$

成立?

如果答案是肯定的, 那么从 (63) 和 (64) 式推知: 与对应于 $P \in \Theta$ 的治疗效果相比, 对应 于 $P=P_{*}$ 的治疗效果是最优的. 因此, 记 $P_{\text {optimal }}:=P_{*}$.

从公式 (62)、最优条件 (64) 和 $P \in \Theta$ 推得

$$
\bar{P}_{\text {optimal }} \equiv 1-P_{\text {optimal }}=\frac{\mu}{\delta},
$$

其中假设 (62) 中除 $P$ 以外的参数都是给定的. 证毕.

下面, 我们数值的研究模型 (22)-(31). 我们用如下典型的参数值 (参见文献 $[15,21,22]$ ):

$$
\begin{aligned}
& \eta=1.2, a=2, q_{0}=0.5, \lambda=3.2 \times 10^{-4}, \mu=1 / 72, \\
& \delta=1 / 48, \gamma=1, p_{0}=3.73, D=1, R(0)=1 .
\end{aligned}
$$

对以上参数值, 定理 8.1 中的 $P_{\mathrm{optimal}}$ 和 $\left(x_{*}, y_{*}, v_{*}\right)$ 取以下近似值:

$$
P_{\mathrm{optimal}} \approx 0.333 ; \quad\left(x_{*}, y_{*}, v_{*}\right) \approx(0.0183,0.1178,0.0016) .
$$

如果初始的 $(x, y, v)$ 接近 $\left(x_{*}, y_{*}, v_{*}\right)\left(\left(x_{*}, y_{*}, v_{*}\right)\right.$ 随 $P \in \Theta$ 而变化), 那么定理 8.1 找到了 一个最优的抑制剂剂量. 我们也问下列一个问题:

给定一般的不依赖于 $P(0 \leqslant P \leqslant 1)$ 的初始数据 $\left(x_{0}, y_{0}, v_{0}\right)$, 是否存在一个 $P_{\mathrm{optimal}}$ 使得

$$
\left.\left.R(t)\right|_{P=P_{\text {optimal }}}=\min _{0 \leqslant P \leqslant 1} R(t) \quad \text { (对大的 } t\right)
$$

成立 (这里假设模型 $(22)-(31)$ 中除 $P$ 以外的参数都是给定的)?

根据我们的数值模拟 (如图 1), 对上述问题有部分答案: 当初值 $\left(x_{0}, y_{0}, v_{0}\right)$ 落在某些范 围之内时, 存在最优的 $P_{\text {optimal }}$.

图 1 显示了不同的抑制剂剂量对肿瘤生长的影响, 其中取 $\left(x_{0}, y_{0}, v_{0}\right)=(0.02,0.15,0.002)$. 与上面的解析结果 (65) 比较, 我们的数值模拟表明: 最优的抑制剂量依赖于初始密度 $\left(x_{0}, y_{0}\right.$, $v_{0}$ ). 为了取得好的治疗效果, 病毒感染的促进与病毒复制的抑制之间必须取得一个合理的平 衡, 因而应聪明的选取 MEK 抑制剂剂量. 


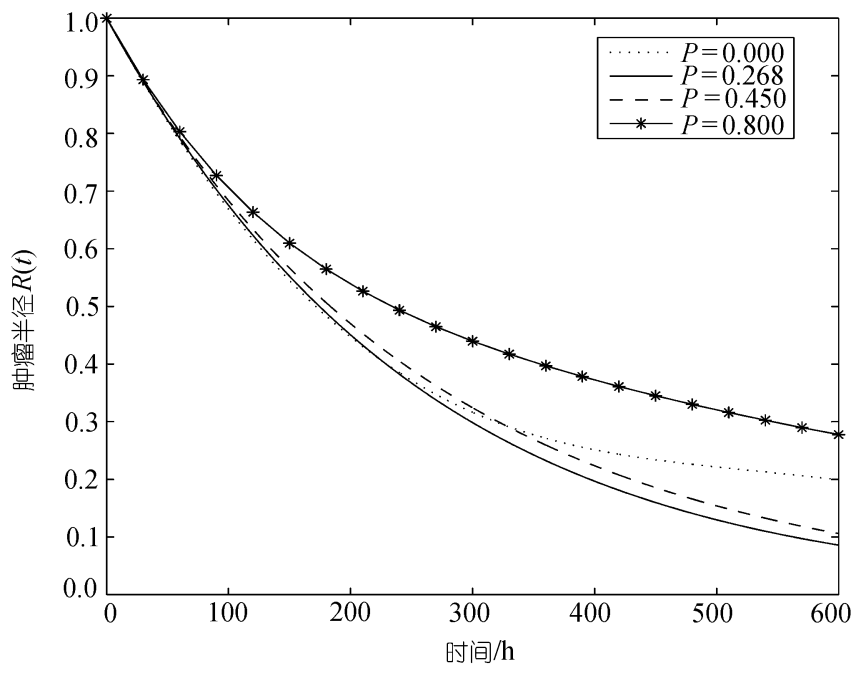

图 1 不同的 MEK 抑制剂剂量对肿瘤生长的影响. 模拟表明: $\boldsymbol{P}_{\text {optimal }} \approx 0.268$

\section{9 最优的抑制剂实施时间}

本节数值的研究不同的 MEK 抑制剂实施时间对肿瘤生长的影响. 在系统 (22)-(31) 中, 取 $\left(x_{0}, y_{0}, v_{0}\right)$ 接近 $\left(x_{*}, y_{*}, v_{*}\right), P(t) \equiv 0$ 当 $0 \leqslant t<t_{m}$ 时; $P(t) \equiv 1-\mu / \delta$ 当 $t_{m} \leqslant t \leqslant T$ 时 (这里 $t_{m}$ 表示当在初始时刻注射病毒后, 经过时间 $t_{m}$, 再注射 MEK 抑制剂). 我们的数 值模拟 (图 2) 显示: 最优的 MEK 抑制剂实施时间 $t_{m}^{\mathrm{opt}}=0$. 即, 为了取得好的肿瘤治疗 效果, 应该同时注射病毒和抑制剂. 在图 2 中, 我们取 $P=0.3333 \sim P_{\text {optimal }}=1-\frac{\mu}{\delta}$ 和 $\left(x_{0}, y_{0}, v_{0}\right)=(0.018,0.118,0.0016) \sim\left(x_{*}, y_{*}, v_{*}\right)$.

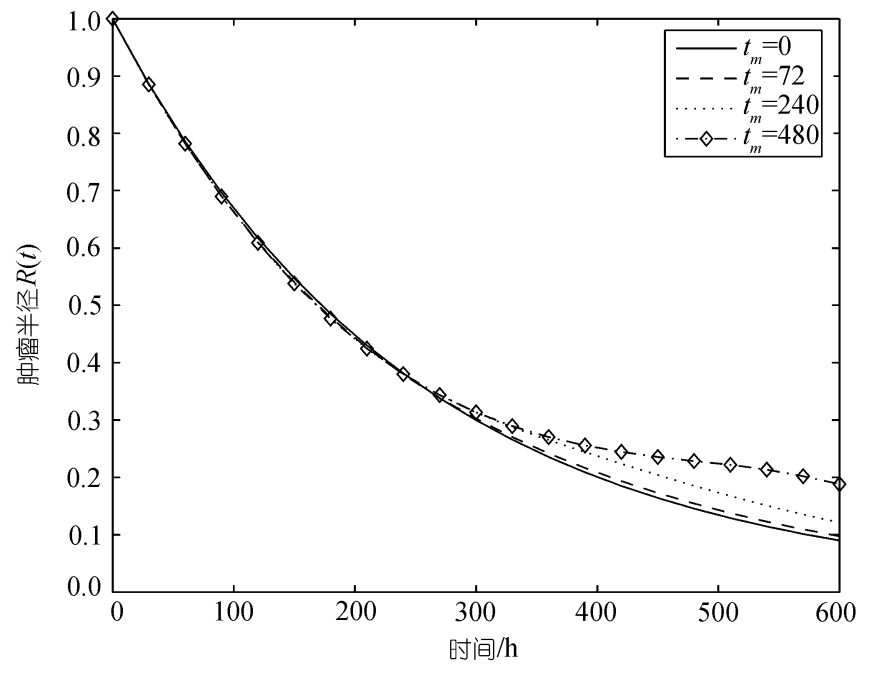

图 2 不同的 MEK 抑制剂实施时间对肿瘤生长的影响. 最优实施时间 $t_{m}^{\mathrm{opt}}=0$

\section{0 小结}

许多自由边界问题来源于物理 (例见文献 [22] 及其所引用的参考文献). 然而, 当今一些 
新的自由边界问题来源于生物. 本文发展了一个自由边界模型来描述病毒和 MEK 抑制剂对 肿瘤生长的影响. 该模型可看成已有的 ODE 模型 ${ }^{[13]}$ 的推广. MEK 抑制剂能刺激 CAR 的 表达, 从而增加病毒的感染. 然而, MEK 抑制剂也能引起短暂的 G1 细胞循环停止, 从而抑制 病毒的复制, 因此, 抑制剂的剂量必须聪明的操纵. 我们的数学模型是一个偏微分方程系统的 自由边界问题. 未知量有: 肿瘤半径, 未被感染的瘤细胞、感染的瘤细胞、坏死的瘤细胞密度, 细胞外病毒密度, Coxsackie 腺病毒受体 (CAR) 的表达水平和细胞的运动速度. 本文的模型 也可以视为已有的肿瘤病毒治疗的 PDE 模型 ${ }^{[11]}$ 的推广. 本文考虑了用病毒和抑制剂综合 治疗肿瘤的数学模型.

对任何抑制剂强度 $P(0 \leqslant P<1)$, 我们能找到一对初始密度

$$
x(r, 0) \sim \text { 常数 }=x_{s}, \quad y(r, 0) \sim \text { 常数 }=y_{s},
$$

使得下面的结论成立 (见定理 6.1 和 7.1):

在生物合理范围内, 任给一个初始半径 $R(0)$, 通过注射以下密度的病毒

$$
v(r, 0) \sim \text { 常数 }=v_{s} \quad \text { 其中 } v_{s}=\frac{\delta}{\gamma}(1-P) y_{s},
$$

并操纵 MEK 抑制剂的剂量为 $P$, 就能使肿瘤半径 $R(t)$ 指数单调减少趋向于零 (当 $t$ 趋于 $\infty$ 时).

我们进一步研究了可能的最优 MEK 抑制剂的剂量 (本文没有考虑病毒和抑制剂的副作 用). 数学分析 (定理 8.1) 表明: 为取得一个好的肿瘤治疗效果, 可以先操纵初始密度

$$
x(r, 0) \sim x_{*}, \quad y(r, 0) \sim y_{*}
$$

(这里 $\left(x_{*}, y_{*}\right)$ 由 $(42)$ 式给出, 其中 $\left.\bar{P}=\mu / \delta\right)$, 然后操纵 MEK 抑制剂的剂量为 $P \sim 1-\mu / \delta$, 并注射密度为 $v(r, 0) \sim \delta \bar{P} y_{*} / \gamma$ 的病毒.

我们也数值的研究可能的最优的 MEK 抑制剂实施时间. 数值结果显示: 为取得好的肿 瘤治疗效果, 应该同时注射病毒和抑制剂.

既然化疗和放疗广泛用于癌症治疗, 那么一些模型推广可能是有趣的. 例如, 考虑化疗和 病毒治疗的组合治疗模型将是一个有意义的问题; 考虑化疗、放疗和病毒治疗的组合治疗模 型将是另一个有意义的问题.

\section{参考文献}

1 Adam J, Bellomo N. A Survey of Models for Tumor-Immune System Dynamics. Boston, MA: Birkhäuser, 1997

2 Bellomo N, Forni G. Looking for new paradigms towards a biological-mathematical theory of complex multicellular systems. Math Models Methods Appl Sci, 16: 1001-1029 (2006)

3 Bertuzzi A, Fasano A, Gandolfi A. A mathematical model for tumor cords incorporating the flow of interstitial fluids. Math Models Methods Appl Sci, 15: 1735-1778 (2005)

4 Chaplain M A J, Lolas G. Mathematical modelling of cancer cell invasion of tissue: The role of the urokinase plasminogen activation system. Math Models Methods Appl Sci, 15: 1685-1734 (2005)

5 Dingli D, Cascino M D, Josić K, et al. Mathematical modeling of cancer radiovirotherapy. Math Biosci, 199: 80-103 (2006)

6 Jackson T L, Byrne H M. A mathematical model to study the effects of drug resistance and vasculature on the response of solid tumors to chemotherapy. Math Biosci, 164: 17-38 (2000)

7 Ward J P, King J R. Mathematical modelling of drug transport in tumour multicell spheroids and monolayer cultures. Math Biosci, 181: 177-207 (2003) 
8 Wodarz D. Viruses as antitumor weapons: defining conditions for tumor remission. Cancer Res, 61: 35013507 (2001)

9 Wu J T, Byrne H M, Kirn D H, et al. Modeling and analysis of a virus that replicates selectively in tumor cells. Bull Math Biol, 63: 731-768 (2001)

10 Jain R. Barriers to drug delivery in solid tumors. Sci Am, 271: 58-65 (1994)

11 Friedman A, Tao Y. Analysis of a model of a virus that replicates selectively in tumor cells. J Math Biol, 47: 391-423 (2003)

12 Bergelson J M, Cunningham J A, Droguett G, et al. Isolation of a common receptor for Coxsackie B viruses and adenoviruses 2 and 5. Science, 275: 1320-1323 (1997)

13 Zurakowski R, Wodarz D. Model-driven approaches for in vitro combination therapy using ONYX-015 replicating oncolytic adenovirus. J Theoret Biol, 245: 1-8 (2007)

14 Friedman A, Tian J P, Fulci G, et al. Glioma virotherapy: Effects of innate immune suppression and increased viral replication capacity. Cancer Res, 66: 2314-2319 (2006)

15 Tao Y, Guo Q. The competitive dynamics between tumor cells, a replication-competent virus and an immune response. J Math Biol, 51: 37-74 (2005)

16 Norris E S, King J R, Byrne H M. Modelling the response of spatially structured tumours to chemotherapy: Drug kinetics. Math Comput Modelling, 43: 820-837 (2006)

17 Tao Y, Chen M. An elliptic-hyperbolic free boundary problem modelling cancer therapy. Nonlinearity, 19: 419-440 (2006)

18 Tao Y, Guo Q. A free boundary problem modelling cancer radiovirotherapy. Math Models Methods Appl Sci, 17: 1241-1259 (2007)

19 Tao Y, Yoshida N, Guo Q. Nonlinear analysis of a model of vascular tumour growth and treatment. Nonlinearity, 17: 867-895 (2004)

20 Tao Y, Zhang H. A parabolic-hyperbolic free boundary problem modelling tumor treatment with virus. Math Models Methods Appl Sci, 17: 63-80 (2007)

21 Wu J T, Kirn D H, Wein L M. Analysis of a three-way race between tumor growth, a replication-competent virus and an immune response. Bull Math Biol, 66: 605-625 (2004)

22 Yi F, Tao Y, Liu Z. Quasi-stationary Stefan problem as limit case of Mullins-Sekerka problem. Sci China Ser A-Math, 40(2): 151-162 (1997) 